\title{
Interactive comment on "Environmental control of natural gap size distribution in tropical forests" by Youven Goulamoussène et al.
}

\author{
M. Bauters (Referee) \\ marijn.bauters@ugent.be
}

Received and published: 17 October 2016

In this manuscript Goulamoussène et al. report on an interesting analysis on the environmental drivers behind gap size distribution in tropical forests, using Lidar data. Their methodology builds on previous work by Lobo and Dalling (2014), but they introduce an interesting new method to determine height thresholds and minimum gap sizes. They rightfully criticize the empirical cutoffs that have been used, and come up with an innovative and interesting alternative. The authors further introduce Lidar-derived environmental parameters to include in the Bayesian model, which enables them to assess the effect of physical environment on gap size distribution. prove/clarify their manuscript in some parts; both for science and form/language. 
A first point would be the height cutoff; the whole study is dependent on the value you set here. You set up a nice probabilistic model, but then you still go back to an empirical threshold (being 0,001percentile). If you have no theoretical considerations to justify this cutoff, than your method is as such not better than any other empirical threshold used in previous studies. Hence, this would need bit more clarification. I wonder whether you could not use the average of the second (lower) normal distribution as a cutoff. This would, I believe, also increase the applicability of your method in other forest types/regions, since the relative difference between canopy tree height/gap height will shift in other forest types. Even if you have a sound reason to use 0,001perc in this case, you would need to reselect a threshold in other forest types.

Secondly, I have not been working with Bayesian statistics myself, but I think the manuscript should be clear for the broad readership of Biogeosciences. Some questions related to the rest of your methodology: -I wonder why you use equation 8 to constrain lambda. What is the reasoning behind an exponential model? -You explain the interpretation of Lambda on $p 5 L$ 146-150. I think the authors are confused here (or maybe I am...); "lambda is not defined when lambda $>=1$ ?? ", and "A value close to 1 means there are a large number of small gaps". Both of these statements seem absolutely wrong to me (I would expect the contrary with both), and they are actually vital to the interpretation of this very manuscript. Either I am wrong, but if not, I am a bit worried for the misinterpretation of the results by the authors. Please have a look at this. Maybe this is also at the basis for the contradiction in some of the statements through the manuscript; Abstract (L10); “... with large gaps being more frequent on hillslopes". Discussion (L296-297): "We found similar results to Lobo and Dalling in $\mathrm{BCl}$; i.e., large gaps are more frequent on gentle slopes". Conclusion: "We expected that slope would also play an important role, with steeper slopes leading to larger gap sizes, but found the opposite effect." Please go through the MS again and make sure the interpretations are the same, and are right, everywhere. If not, you fail to give the reader a clear take home message...

Printer-friendly version

Discussion paper 
Thirdly, I have listed some other comments. The list is not complete; some of these are clear typos or sloppiness. This would need to be avoided for your resubmission... In general; make sure results, $\mathrm{M}$ and $\mathrm{M}$, and discussion are in the appropriate section, redo your subheadings, avoid typos, avoid repetition...

-P1 L10: "we plan to scale up"

-P2 L23: a large quantity of leaf and wood litter becomes available. But please rephrase this anyway. It's not a good sentence. Mineralisation and decomposition makes the nutrients available, not the wood and leaf litter available as such.

-p4 L90: the buffer you applied to anthropogenic tracks: is the Approuage an anthropogenic track? For sure not masking out natural rivers out of your algorithm would hugely affect your results. I think (hope) you did include natural rivers in your buffers, but you would need to rephrase, since these are not anthropogenic...

P4: Sloppiness; your subtitles have the same rank on this page, while they all fall within the first subtitle 'Environmental data'

P5 L 141-142: what do you define as contiguous? Diagonal pixels would be contiguous? You know from the field that some trees may be left standing in certain gaps, so this could be important for your results...

P6 I 153-154: Please cite both R packages properly.

P6 L172: Here you use $X$ as the vector of covariates; while on the next page in equation 8 you use varig. Please be consistent to make your MS more comprehensible.

P7 L191-193: For clarity I would rename the transformed variables. Also in eq 6 you use the HA as the new variable, and the hydraulic altitude in full as the old, while in 2.1.6. on $\mathrm{p} 4$ you already use the $\mathrm{HA}$ abbreviation. Please correct these small errors for your future readers.

P7 I 204: investigated; Material and methods should be in past tense. Please correct

Printer-friendly version

Discussion paper 
everywhere.

Figure 4: This figure does not have a lot of information. I would leave it out and describe in text instead.

P14 L285: why don't you show the values from the Kellner studies in brackets, like you do for Lobo and Asner?

P14 L300: Please add reference to your $75 \%$ statement. . .

P 14 I 309-311: "Together with. .." I don't get this sentence. Please rephrase...

P 15 I 330-331: Really? The first study? And what about Lob and Dallin (a study which served as a basis for your study)

Please review the reference list.

Interactive comment on Biogeosciences Discuss., doi:10.5194/bg-2016-320, 2016. 